Document downloaded from:

http://hdl.handle.net/10251/52708

This paper must be cited as:

Cantó Colomina, B.; Coll, C.; Sánchez, E. (2014). Stability robustness of perturbed structured systems. Journal of The Franklin Institute. 351(3):1195-1204. doi:10.1016/j.jranklin.2013.11.020.

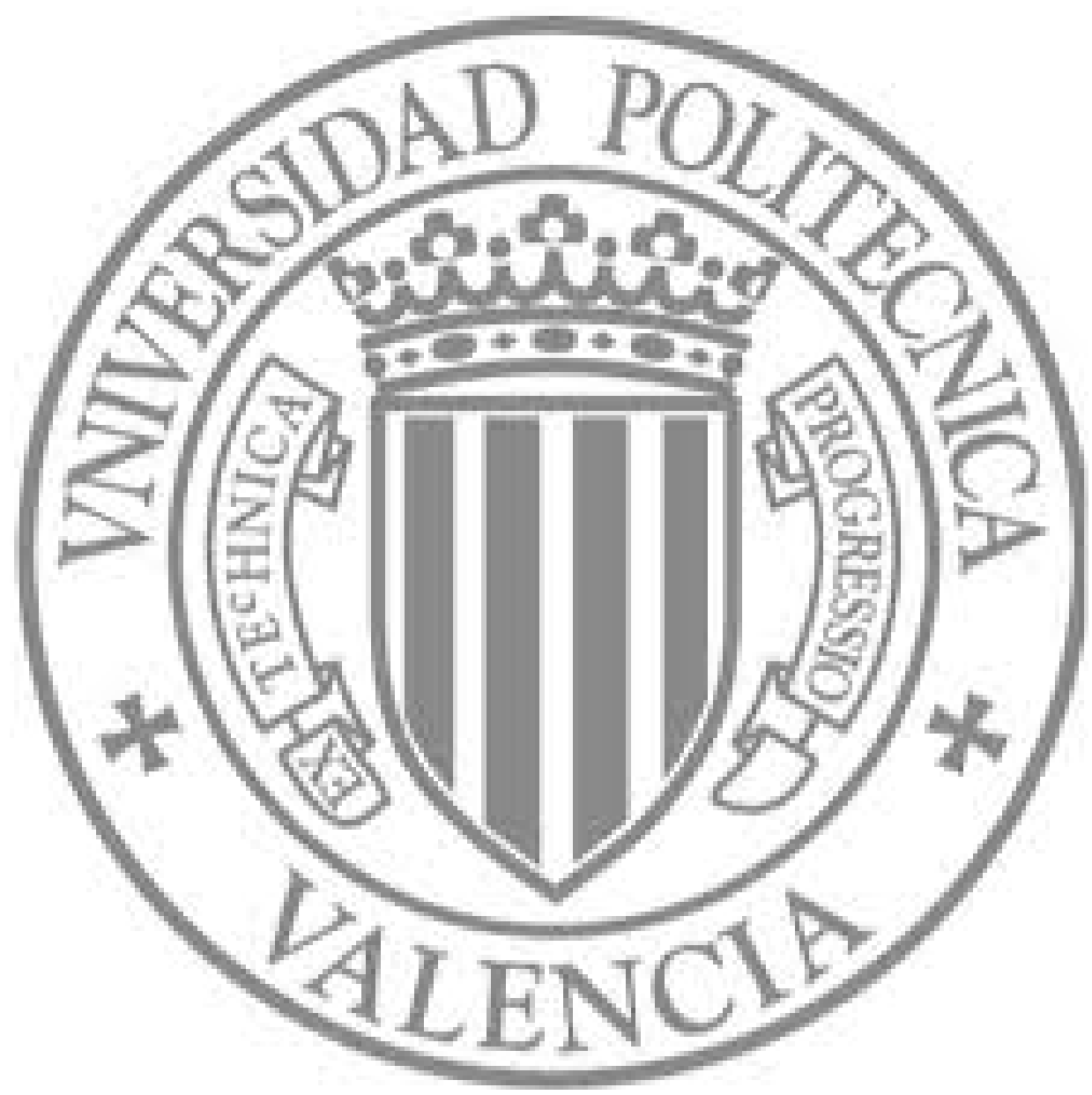

The final publication is available at

http://dx.doi.org/10.1016/j.jfranklin.2013.11.020

Copyright Elsevier 


\title{
Stability robustness of perturbed structured systems
}

\author{
Begoña Cantó, Carmen Coll, Elena Sánchez \\ Institut de Matemàtica Multidisciplinar \\ Departament de Matemàtica Aplicada \\ Universitat Politècnica de València \\ Camino de Vera, 14, 46071, València, Spain \\ \{bcanto,mccoll,esanchezj\}@mat.upv.es
}

\begin{abstract}
In this paper, the robust stability problem of structured linear systems is analyzed. In order to preserve the structure of the initial system, the structure of the admissible perturbations is characterized. Frobenius and infinity matrix norms are used to study the robust stability of the system affected by different admissible perturbations. Upper bounds for the perturbations are obtained, which guarantee that the perturbed system remains stable. Finally, some examples are shown to illustrate the results.
\end{abstract}

\section{Introduction}

Robust stability is an important requirement for analysis and design of control systems. Usually, robust stability problems appear in the engineering modeling where it is important to know the behavior of the system under small perturbations and to ensure that the system remains stable for the full range of possible perturbations. Efficient mathematical approach to treat robust stability problems is given in [1]. A typical question in solving these problems is to determine the "distance to instability" of a given system under perturbations. Commonly, this solution involves the eigenvalues of the coefficient matrix. 
In the last twenty years, this topic was studied by several authors and some bounds on perturbations guaranteeing the stability of the system have been obtained. Different methods exist to study the robustness problem. For instance, the stability radius concept has been used to study the robust stability of state-space models in $[2,3,4]$. However, the stability radius concept may be too conservative. The idea to construct Lyapunov functions to study robust stability has attracted considerable attention. In this way, some results on robust stability are given in [5] and in [6] robust stability bounds can be derived and it has been shown that these bounds are less conservative in most cases that the existing ones. The robust Schur and Hurwitz stability of the interval systems are considered in [7] and output feedback stabilization conditions and robust stability with respect to the delay time uncertainty are given in [8].

Usually, the mathematical formulation of a model is given by means of structured matrices. Then, the robust stability consists in finding conditions to guarantee desired performances in presence of structured perturbations. Among the extensive literature on robust stability we focus on discrete-time system. In [9] sufficient robust stability conditions is given in terms of linear matrix inequalities (LMI)s, the idea is to introduce new variables such that the existence of a parameter dependent Lyapunov function is guaranteed. In the same way, in [10] a robust stability condition and a new state feedback stabilizability LMI problem based on this condition are provided. More recently, an improved condition for robust D-stability has been given in [7].

When a finite-difference method is applied to solve a partial differential equation the associated discrete model obtained shows structured matrices, see [11]. Motivated by the structure of the discrete approximation and the possibility that the coefficient matrices are subjected to some perturbations, in this paper, bounds are obtained assuming only perturbation with a fixed structure. The approach is based on some properties of the spectrum of tridiagonal matrices. We will focus on the localization of the eigenvalues of such matrices and we will intend to exploit the specific structure of the matrix to obtain bounds whose do not depend on complex calculations. This structural requirement may seem to bring restrictions to the applicability of this method, but in fact, similar structure is presented several mathematical models which have been used and are of potential use in image processing. For example, Jain, in [12], made use of a semicasual image 
model which uses symmetric tridiagonal matrices.

In the classical approach to study robust stability, it is used the Lyapunov theory to construct convenient functions and to estimate the robust stability from each of them. By contrast, our study in the present paper will be focused on the determination of bounds that can be calculated directly from the initial coefficient matrices. This fact is worthwhile to reduce the number of computations in the design of algorithms for solving engineering problems involving robust control.

The paper is structured as follows. In Section 2 a structured system is obtained applying the finite-difference method to a partial differential equation. Some concepts and results used in this paper are introduced. In Section 3 the characteristic polynomial of a tridiagonal matrix is analyzed. Next, in the Section 4 the stability problem is studied when the system is affected by different perturbations and some results on bounds to assure the stability of the perturbed system are given.

\section{Statement of the problem and pre- liminary}

Consider the second order partial differential equation given by

$$
\frac{\partial z}{\partial t}+F(\mathbf{p}) \frac{\partial^{2} z}{\partial x^{2}}+G(\mathbf{p}) z=O
$$

where $F(\mathbf{p}), G(\mathbf{p})$ are matrices in $\mathbf{R}^{n \times n}$ and $\mathbf{p}=(\alpha, \beta) \in \mathbf{R}^{2}$ is an unknown parameter vector. The solution of (1), denoted by $z=$ $z(t, x)$, depends on $\mathbf{p}$, the spatial variable $x \in \Omega$ and the time value $t \in(0, T)$.

The partial differential equation is replaced with a discrete approximation. Both space and time can be discretized by grid with parameter $\Delta x$, the distance between two adjacent nodes of the grid, and parameter $\Delta t$, the local distance between adjacent time steps in the interval $(0, T)$.

In this case, we approximate the time derivative in (1) with a forward difference and the space derivative in (1) with the central difference, we have

$$
\frac{\partial z}{\partial t} \approx \frac{z_{k+1, i}-z_{k, i}}{\Delta t}, \quad \frac{\partial^{2} z}{\partial x^{2}} \approx \frac{z_{k, i-1}-2 z_{k, i}+z_{k, i+1}}{(\Delta x)^{2}} .
$$


Taking $\Delta x=\Delta t=1, F(\mathbf{p})=\alpha I$ and $G(\mathbf{p})=\beta I$, the discretized problem can be written as

$$
\begin{aligned}
z_{k+1, i}= & p z_{k, i}+q\left(z_{k, i-1}+z_{k, i+1}\right) \\
z_{k+1}= & \left(\begin{array}{ccccc}
p & q & 0 & \cdots & 0 \\
q & p & q & \cdots & 0 \\
0 & q & p & \cdots & 0 \\
\vdots & \ddots & \cdots & \ddots & q \\
0 & 0 & \cdots & q & p
\end{array}\right) z_{k}
\end{aligned}
$$

where $p=1+2 \alpha-\beta$ and $q=-\alpha$ (see [11] for more information).

Note that (2) is a particular case of the more general linear time invariant system

$$
x_{k+1}=A_{n+m}(p, q) x_{k}+B(d) u_{k}
$$

where $u(k)$ is the input vector and $x(k)$ is the state vector, with the parameters in the set $\mathcal{P}=\{(p, q, d) / d \neq 0\}$,

$$
B(d)=\left(\begin{array}{c}
O \\
d I_{m}
\end{array}\right)
$$

and

$$
A_{n+m}(p, q)=\left(\begin{array}{cc}
A_{n}(p, q) & \mathcal{A}^{T}(q) \\
\mathcal{A}(q) & A_{m}(p, q)
\end{array}\right)
$$

where $A_{k}(p, q)=\left(a_{i j}\right)$ is a tridiagonal matrix of size $k$ with $a_{i i}=p$ and $a_{i, i+1}=a_{i, i-1}=q$. And $\mathcal{A}(q)$ is a matrix where the only entry nonzero is the $(1, n)$-entry, which is equal to $q$. Moreover, we denote by $\mathcal{M}$ the set of matrices with structure given in (5).

We recall that, a matrix $A$ is stable (also referred to as Schur stable or convergent) if all its eigenvalues lie in the open unit disk of the complex plane, that is, $\rho(A)<1$, where $\rho(\cdot)$ denote the spectral radius of a matrix, see [13]. The stability of a dynamic system (3) is related to the stability of its state coefficient matrix.

Note that the system (3) is a structured system with unknown parameters. Before studying the robust stability problem we need to know the parameters to accurate the model. That is, to identify the unknown parameters uniquely from the response of a considered experiment. This property is known as identifiability problem. Using the Markov parameters associated to the structured system with the 
matrices $B(d)$ and $A_{n+m}(p, q)$ given by (4) and(5), it is easy to check that the system is identifiable, see [14].

In this paper we focus our attention on the robustness of the stability of a perturbed discrete-time system, but the perturbations are restricted to be structure preserving. In general, in order to preserve the structure of the initial system it is necessary to know the structure of the admissible perturbations, and then find a bound of them to assure the robust stability of the problem. Thus, let the parametric linear system be

$$
x_{k+1}=A(\vartheta) x_{k},
$$

where the matrix $A(\vartheta)$ belongs to the set $\mathcal{M}$, being $\vartheta$ the parametric vector. This system is robustly stable if all eigenvalues of matrix $A(\vartheta)$ have magnitudes less than one, that is $\rho(A(\vartheta))<1$, for all the values of $\vartheta$ in the admissible set of parameters.

Throughout the paper we will use some matrix norms. We recall that the infinity matrix norm of a matrix $A=\left(a_{i j}\right)$ of order $l$ is the maximum absolute row sum of the matrix, that is $\|A\|_{\infty}=$ $\max _{1 \leq i \leq l}\left(\sum_{j=1}^{l}\left|a_{i j}\right|\right)$, and the Frobenius matrix norm is given by $\|A\|_{F}=$ $\left(\operatorname{trace}\left(A^{T} A\right)\right)^{\frac{1}{2}}$.

The robust stability problem is to know the impact on the spectrum of matrix $A$ where the matrix is subjected to a perturbation. It is known that if $A$ and $A+\Delta A$ are normal, that is, $A A^{*}=A^{*} A$, then there exists a permutation such that $\|\Lambda-\tilde{\Lambda}\|_{F} \leq\|\Delta A\|_{F}$, being $\Lambda$ and $\tilde{\Lambda}$ the diagonal matrices which entries are the eigenvalues of $A$ and $A+\Delta A$, respectively (Hoffman-Wielandt Theorem).

We begin our discussion by studying the spectrum of a tridiagonal matrix. For that, in the next section, we relate the characteristic polynomial of tridiagonal matrices with different sizes and we give some results on stability. These results are useful to study the characteristic polynomial of a perturbed matrix.

\section{Spectrum of tridiagonal matrices}

In this section, the eigenvalues of the matrix $A_{i}(p, q)$ and the eigenvalues of its submatrices $A_{j}(p, q), j=1, \ldots, i$ are related. We denote $d_{0}(z)=1, d_{1}(z)=z-p$ and $d_{j}(z)=\operatorname{det}\left(z I-A_{j}(p, q)\right)$ and $\sigma\left(A_{j}(p, q)\right)=\left\{\lambda_{k}^{j}, k=1, \ldots, j\right\}, j=1, \ldots i$. It is known that the 
collection of these polynomials satisfies the Sturm sequence

$$
d_{j}(z)=d_{j-1} d_{1}(z)-q^{2} d_{j-2}(z) .
$$

Moreover, the spectrum of two consecutive submatrices are related as follows

$$
\lambda_{1}^{j}<\ldots \lambda_{k-1}^{j}<\lambda_{k-1}^{j-1}<\lambda_{k}^{j}<\ldots<\lambda_{j}^{j} .
$$

Thus, $d_{j}(z)$ and $d_{j-1}(z)$ have not common roots. For more details see reference [15].

In the next proposition the transmission of the stability for matrices, with the structure given in (5), depending on its size is discussed. So, if a matrix of size $i$ is stable, any matrix whose size is less than $i$ will also be stable. If a matrix of size $i$ is not stable any matrix of size larger than $i$ will not be stable.

\section{Proposition 1.}

a) If $A_{i}(p, q)$ is stable then $A_{j}(p, q)$ is stable, for all $j<i$.

b) If $A_{i}(p, q)$ is not stable then $A_{j}(p, q)$ is not stable, for all $j \geq i$.

Some properties are given in the next proposition.

\section{Proposition 2.}

a) $d_{2 j}(z)=d_{j}^{2}(z)-q^{2} d_{j-1}^{2}(z)$.

b) The only common root of $d_{2 j+1}$ and $d_{2 j-1}$ is $z=p$.

c) $d_{2 j}(z)$ and $d_{2 j-2}(z)$ have not common roots.

Proof. a) We prove this property by induction hypothesis. It is straightforward for $j=1$. Suppose that the property holds on to $j-1$ then, using the Sturm sequence we have

$$
\begin{aligned}
& d_{2 j}(z)=d_{2 j-1}(z) d_{1}(z)-q^{2} d_{2 j-2}(z)= \\
& \quad=\left(d_{2 j-2}(z) d_{1}(z)-q^{2} d_{2 j-3}(z)\right) d_{1}(z)-q^{2} d_{2 j-2}(z) \\
& =d_{2 j-2}(z) d_{1}^{2}(z)-q^{2}\left(d_{2 j-2}(z)+q^{2} d_{2 j-4}(z)\right)-q^{2} d_{2 j-2}(z) \\
& =d_{2 j-2}(z)\left(d_{1}^{2}(z)-2 q^{2}\right)-q^{4} d_{2 j-4}(z) \\
& =\left(d_{j-1}^{2}(z)-q^{2} d_{j-2}^{2}(z)\right)\left(d_{1}^{2}(z)-2 q^{2}\right)-q^{4}\left(d_{j-2}^{2}(z)-q^{2} d_{j-3}^{2}(z)\right) .
\end{aligned}
$$


On the other hand,

$$
\begin{aligned}
d_{j}^{2}(z)- & q^{2} d_{j-1}^{2}(z)= \\
= & \left(d_{j-1}(z) d_{1}(z)-q^{2} d_{j-2}(z)\right)^{2}-q^{2}\left(d_{j-2}(z) d_{1}(z)-q^{2} d_{j-3}(z)\right)^{2} \\
= & d_{j-1}^{2}(z) d_{1}^{2}(z)-2 q^{2} d_{j-1}(z)\left(d_{j-1}+q^{2} d_{j-3}\right)+q^{4} d_{j-2}^{2}(z) \\
& -q^{2} d_{j-2}^{2}(z) d_{1}^{2}(z)+2 q^{4} d_{j-2}(z) d_{1}(z) d_{j-3}(z)-q^{6} d_{j-3}^{2}(z) \\
= & d_{j-1}^{2}(z)\left(d_{1}^{2}(z)-2 q^{2}\right)-q^{2} d_{j-2}^{2}(z)\left(d_{1}^{2}(z)-q^{2}\right) \\
& +2 q^{4} d_{j-3}(z)\left(d_{j-2} d_{1}-d_{j-1}\right)-q^{6} d_{j-3}^{2}(z) \\
= & \left(d_{j-1}^{2}(z)-q^{2} d_{j-2}^{2}(z)\right)\left(d_{1}^{2}(z)-2 q^{2}\right)-q^{4}\left(d_{j-2}^{2}(z)-q^{2} d_{j-3}^{2}(z)\right) .
\end{aligned}
$$

Thus, the property is shown.

b) We observe that $d_{1}(p)=0$ and $d_{3}(p)=0$. Suppose that $d_{2 j-1}(p)=0$, then by (6) $p$ is also root of $d_{2 j+1}(z)$. On the other hand, if $a$ satisfies $d_{2 j+1}(a)=d_{2 j-1}(a)=0$, then $d_{1}(a) d_{2 j}(a)=0$. Hence $a=p$, since $d_{2 j}(z)$ and $d_{2 j-1}(z)$ have not common roots.

c) Now, we suppose that $a$ satisfies $d_{2 j}(a)=d_{2 j-2}(a)=0$. Then $d_{1}(a) d_{2 j-1}(a)=0$. This is a contradiction since $d_{2 j}(z)$ and $d_{2 j-1}(z)$ have not common roots.

\section{Robust stability problem}

In the following result we establish a bound which preserves the stability of the perturbed matrix.

Proposition 3. Consider the stable matrix $A_{n+m}(p, q)$ and a normal perturbed matrix $\widetilde{A}_{n+m}=A_{n+m}(p, q)+\Delta A$. If $\|\Delta A\|_{F}<1-$ $\rho\left(A_{n+m}(p, q)\right)$, then $\widetilde{A}_{n+m}$ is also stable.

Proof. Denote $\rho=\rho\left(A_{n+m}(p, q)\right)$. Using the Hoffman-Wielandt Theorem, there exists a permutation such that $\|\Lambda-\tilde{\Lambda}\|_{F} \leq\|\Delta A\|_{F}<1-\rho$, being $\Lambda=\operatorname{diag}\left(\lambda_{i}\right)$ and $\tilde{\Lambda}=\operatorname{diag}\left(\tilde{\lambda}_{i}\right)$ the diagonal matrices which entries are the eigenvalues of $A_{n+m}(p, q)$ and $\widetilde{A}_{n+m}$, respectively. Hence, for all $i$,

$$
\left|\tilde{\lambda}_{i}\right| \leq\left|\tilde{\lambda}_{i}-\lambda_{i}\right|+\left|\lambda_{i}\right|<1-\rho+\left|\lambda_{i}\right| \leq 1 .
$$

Thus, $\widetilde{\rho}=\rho\left(\widetilde{A}_{n+m}\right)<1$.

To illustrate the usefulness of the results of this paper we consider a practical system. 
Example 1. Consider the heat equation given by

$$
\frac{\partial z}{\partial t}-\frac{1}{3} \frac{\partial^{2} z}{\partial x^{2}}=O
$$

which solution $z=z(t, x)$, depends on the spatial variable $x \in[0,3]$ and the time value $t \in(0, T)$. In the same way that Section 2 and considering a control on the last grid node, with $d=1$, the discretized problem is given by

$$
z_{k+1}=A_{3}\left(\frac{1}{3}, \frac{1}{3}\right) z_{k}+B(1) u_{k} .
$$

Note that the system is stable since $\rho=0.8047<1$.

We consider the perturbation $\Delta=\left(\begin{array}{lll}0 & 10^{-2} & 10^{-2}\end{array}\right)$. The perturbed matrix $\widetilde{A}_{3}=A_{3}\left(\frac{1}{3}, \frac{1}{3}\right)+\Delta A$ with $\Delta A=B(1) \Delta$ is a normal matrix. Moreover, $\|\Delta A\|_{F}=0.0141<0.1953=1-\rho$. Hence, the perturbed matrix $\widetilde{A}_{3}$ is also stable with $\widetilde{\rho}=0.6666<1$.

Next, in order to study the effect of a perturbation on the state coefficient matrix of the system (3) we assume that the matrix $A_{n+m}(p, q)$ is subjected to a perturbation

$$
A_{n+m}(p, q) \rightarrow \widetilde{A}_{n+m}=A_{n+m}(p, q)+B(d) \Delta
$$

where $\Delta$ is a structured perturbation matrix defined as

$$
\Delta=\left(\Delta_{1} \Delta_{2}\right), \text { with } \Delta_{1}=\mathcal{A}(\gamma), \Delta_{2}=A_{m}(\alpha, \beta),
$$

where $\alpha, \beta, \gamma$ are unknown nonzero parameters.

By choosing different perturbations we can study different structures for the matrix $\widetilde{A}_{n+m}$. We are interested to know if the stability is preserved under different types of perturbations and to know a bound for the admissible set of perturbations.

It is well-known, [16], that the spectrum of a matrix $A_{l}(p, q) \in \mathcal{M}$ is given by

$$
\sigma\left(A_{l}(p, q)\right)=\left\{\mu_{k}(l)=p+2 q \cos \frac{k \pi}{l+1}, \quad k=1, \ldots, l\right\} .
$$

From now on, we denote $\rho=\rho\left(A_{n+m}(p, q)\right)$ and $\widetilde{\rho}=\rho\left(\widetilde{A}_{n+m}\right)$. 


\subsection{Case 1}

Consider the discrete-time stable system (3). We want to obtain conditions for robust stability.

In this first case we consider a perturbation $\Delta=\left(\Delta_{1} \Delta_{2}\right)$ given in (8) such that the new matrix has the following structure

$$
\widetilde{A}_{n+m}=\left(\begin{array}{cc}
A_{n}(p, q) & \mathcal{A}^{T}(q) \\
O & A_{m}(p+d \alpha, q+d \beta)
\end{array}\right) .
$$

The characteristic polynomial of $\widetilde{A}_{n+m}$ is given by

$$
\operatorname{det}\left(z I-\widetilde{A}_{n+m}\right)=\delta_{m}(z) d_{n}(z)
$$

being $\delta_{0}(z)=1, \delta_{1}(z)=z-p-d \alpha$, and $\delta_{j}(z)=\operatorname{det}\left(z I-A_{j}(p+\right.$ $d \alpha, q+d \beta)), \forall j=1, \ldots, m$.

Let $m=1$ be. In the following result we show that the eigenvalues of the original system are perturbed, except in finite number cases.

Proposition 4. Consider the stable system (3) and the perturbed matrix (10) with $m=1$. If $\lambda \in \sigma\left(A_{n+1}(p, q)\right)$ then $\lambda \notin \sigma\left(\widetilde{A}_{n+1}\right)$, except if $\alpha$ is in the set $\left\{\frac{2 q}{d} \cos \frac{k \pi}{n+2}, k=1, \ldots, n+1\right\}$.

Proof. If $\lambda \in \sigma\left(A_{n+1}(p, q)\right)$, then $\lambda \notin \sigma\left(A_{n}(p, q)\right)$, so $d_{n}(\lambda) \neq 0$. On the other hand, $\operatorname{det}\left(z I-\widetilde{A}_{n+1}\right)=\delta_{1}(z) d_{n}(z)=d_{n}(z)(z-p-d \alpha)$. Thus, $\lambda \notin \sigma\left(\widetilde{A}_{n+1}\right)$, except if $\lambda=p+d \alpha$. By (9) it is only true when $\alpha$ is in the set $\left\{\frac{2 q}{d} \cos \frac{k \pi}{n+2}, k=1, \ldots, n+1\right\}$.

Example 2. Consider the heat equation (7) given in the Example 1 and the perturbation $\Delta=\left(\begin{array}{lll}0-q d \alpha\end{array}\right)=\left(\begin{array}{ll}0 & -\frac{1}{3}-\frac{1}{3}\end{array}\right)$. Note that, $\sigma\left(A\left(\frac{1}{3}, \frac{1}{3}\right)\right)=\{0.8047,0.3333,-0.138\}$ and $\sigma\left(\widetilde{A}_{3}\right)=\{0.6666,0,0\}$. Moreover, $\alpha=-\frac{1}{3}$ is not in the set

$$
\left\{\frac{2 q}{d} \cos \frac{k \pi}{4}, k=1,2,3\right\}=\left\{\frac{\sqrt{2}}{3}, 0,-\frac{\sqrt{2}}{3}\right\} .
$$

Hence the Proposition 4 holds since all eigenvalues of the original matrix have been perturbed. 
On the other hand, if we consider the control $B(d)$ with $d=\sqrt{2}$, then $\Delta=\left(0-\frac{1}{3}-\frac{\sqrt{2}}{3}\right)$ and $\sigma\left(\widetilde{A}_{3}\right)=\{0.6666,-0.138,0\}$. We observe that the eigenvalue -0.138 is also eigenvalue of the original matrix. In this case, an eigenvalue is not perturbed since $\alpha=\frac{1}{3}$ belongs to the set $\left\{\frac{2 q}{d} \cos \frac{k \pi}{4}, k=1,2,3\right\}=\left\{\frac{1}{3}, 0,-\frac{1}{3}\right\}$.

Next, we give conditions to guarantee the stability of the perturbed system using Frobenius norm and infinity norm, respectively.

Proposition 5. Consider the stable system (3) and the perturbed matrix (10). If $\rho<1-q$ and $\left\|\Delta_{2}\right\|_{F}^{2}<\frac{(1-\rho)^{2}-q^{2}}{d^{2}}$, then the perturbed system is stable.

Proof. Note that in this case, $\Delta A=B(d)\left(\mathcal{A}\left(-\frac{q}{d}\right), \Delta_{2}\right)$. Using $\left\|\Delta_{2}\right\|_{F}^{2}<$ $\frac{(1-\rho)^{2}-q^{2}}{d^{2}}$, we obtain

$$
\|\Delta A\|_{F}^{2}=d^{2}\left(\frac{q^{2}}{d^{2}}+\left\|\Delta_{2}\right\|_{F}^{2}\right)<(1-\rho)^{2} .
$$

Hence, the condition established in Proposition 3 holds and we have guaranteed the stability of the perturbed system.

Proposition 6. Consider the stable system (3) and the perturbed matrix (10). If $\left\|\Delta_{2}\right\|_{\infty}<\frac{1-\left|\mu_{1}(m)\right|}{|d|}$, then the perturbed system is stable.

Proof. In this case $\sigma\left(\widetilde{A}_{n+m}\right)=\sigma\left(A_{n}(p, q)\right) \cup \sigma\left(A_{m}(p+d \alpha, q+d \beta)\right)$ with

$\sigma\left(A_{m}(p+d \alpha, q+d \beta)\right)=\left\{p+d \alpha+2(q+d \beta) \cos \frac{k \pi}{m+1}, \quad k=1, \ldots, m\right\}$.

Since $\rho<1$ and from Proposition 1 , we have that $\rho\left(A_{n}(p, q)\right)<1$ and $\rho\left(A_{m}(p, q)\right)<1$. That is, $\left|\mu_{k}(m)\right|<1, k=1, \ldots, m$. Note that, $\left|\mu_{k}(m)\right|-\left|\mu_{1}(m)\right| \leq 0$. Moreover, since $\Delta_{2}$ is a symmetric triangular matrix we have

$$
\left\|\Delta_{2}\right\|_{\infty}=|\alpha|+|2 \beta|
$$


Using $\left\|\Delta_{2}\right\|_{\infty}<\frac{1-\left|\mu_{1}(m)\right|}{|d|}$, then the eigenvalues of $A_{m}(p+d \alpha, q+d \beta)$ satisfy

$$
\left|\mu_{k}(m)+d \alpha+2 d \beta \cos \frac{k \pi}{m+1}\right|<\left|\mu_{k}(m)\right|+|d|\left\|\Delta_{2}\right\|_{\infty} \leq 1 .
$$

The following example illustrates the above results.

Example 3. Consider the matrices $A_{3}=A_{3}\left(\frac{1}{6},-\frac{1}{3}\right), B(1)$ and $\Delta=$ (0 $\left.\frac{1}{3} \frac{1}{10}\right)$. Then $\sigma\left(A_{3}\right)=\{-0.3047,0.1667,0.6381\}$, and $\sigma\left(\widetilde{A}_{3}\right)=$ $\{0.5,-0.1667,0.2667\}$. Both matrices are stable, since $\rho=0.638$ and $\widetilde{\rho}=0.5$. It is easy to check that $\left\|\Delta_{2}\right\|_{F}=\left\|\Delta_{2}\right\|_{\infty}=0.1$ and $\mu_{1}(1)=$ 0.2667. And the conditions $\rho<1-q,\left\|\Delta_{2}\right\|_{F}^{2}<\frac{(1-\rho)^{2}-q^{2}}{d^{2}}$ and $\left\|\Delta_{2}\right\|_{\infty}<\frac{1-\left|\mu_{1}(1)\right|}{|d|}$ are satisfied. This fact is in according to Proposition 5 and Proposition 6.

Finally, as $\alpha=\frac{1}{10} \neq-\frac{2}{3} \cos \frac{k \pi}{4}$, with $k=1,2,3$, all the eigenvalues of $A_{3}\left(\frac{1}{6},-\frac{1}{3}\right)$ have been perturbed as established in Proposition 4.

\subsection{Case 2}

We consider the discrete-time system (3) with a perturbation $\Delta=$ $\left(\Delta_{1} \Delta_{2}\right)$ given in (8) such that the perturbed matrix has the following structure

$$
\widetilde{A}_{n+m}=\left(\begin{array}{cc}
A_{n}(p, q) & \mathcal{A}^{T}(q) \\
\mathcal{A}(q) & A_{m}(p+d \alpha, q+d \beta)
\end{array}\right)
$$

In order to analyze the eigenvalues of this matrix (11) we give a relationship between the characteristic polynomial of $A_{n+m}(p, q)$ and $\widetilde{A}_{n+m}$.

Proposition 7. Consider the matrix $\widetilde{A}_{n+m}$ given in (11). Then

$$
\operatorname{det}\left(z I-\widetilde{A}_{n+m}\right)=\delta_{m}(z) d_{n}(z)-q^{2} \delta_{m-1}(z) d_{n-1}(z) .
$$


Proof. Using $-d \alpha=\delta_{1}(z)-d_{1}(z)$ we obtain

$$
\begin{aligned}
& \operatorname{det}\left(z I-\widetilde{A}_{n+m}\right)= \\
&=d_{n}(z)\left|z I-A_{m}(p+d \alpha, q+d \beta)-q^{2} \frac{\operatorname{adj}\left(z I-A_{n}(p, q)\right)}{d_{n}(z)}\right| \\
&=d_{n}(z)\left(\frac{d_{n+1}(z)-d \alpha d_{n}(z)}{d_{n}(z)}\left|z I-A_{m-1}(p+d \alpha, q+d \beta)\right|\right. \\
&\left.-(q+d \beta)^{2}\left|z I-A_{m-2}(p+d \alpha, q+d \beta)\right|\right) \\
&=\left(d_{n+1}(z)-d \alpha d_{n}(z)\right) \delta_{m-1}(z)-(q+d \beta)^{2} \delta_{m-2}(z) d_{n}(z) \\
&= d_{n}(z) \delta_{m}(z)+\delta_{m-1}(z)\left(d_{n+1}(z)-d_{n}(z) d_{1}(z)\right) .
\end{aligned}
$$

Thus, from $(6) \operatorname{det}\left(z I-\widetilde{A}_{n+m}\right)=\delta_{m}(z) d_{n}(z)-q^{2} \delta_{m-1}(z) d_{n-1}(z)$.

In the following result we consider the perturbed system with $m=$ 1 and we give the relationship between the eigenvalues of the original system with the eigenvalues of the perturbed one.

Proposition 8. Consider the stable system (3) and the perturbed matrix (11) with $m=1$. If $\lambda \in \sigma\left(A_{n+1}(p, q)\right)$ then $\lambda \notin \sigma\left(\widetilde{A}_{n+1}\right)$.

Proof. Using the result obtained in Proposition 7 we have

$$
\begin{aligned}
\operatorname{det}\left(z I-\widetilde{A}_{n+1}\right) & =\delta_{1}(z) d_{n}(z)-q^{2} d_{n-1}(z) \\
& =d_{n}(z)(z-p)-q^{2} d_{n-1}(z)-d \alpha d_{n}(z) \\
& =d_{n+1}(z)-d \alpha d_{n}(z) .
\end{aligned}
$$

If $\lambda$ is an eigenvalue of the matrix $A_{n+1}(p, q)$, then $\operatorname{det}\left(\lambda I-\widetilde{A}_{n+1}\right)=$ $-d \alpha d_{n}(\lambda) \neq 0$, since two consecutive submatrices have not common eigenvalues. Thus, $\lambda \notin \sigma\left(\widetilde{A}_{n+1}\right)$.

To guarantee the stability of the perturbed system when the perturbed matrix is given by (11), using Proposition 3, we have the following result, which proof is straightforward.

Proposition 9. Consider the stable system (3) and the matrix (11). If $\left\|\Delta_{2}\right\|_{F}<\frac{1-\rho}{|d|}$, then the perturbed system is stable.

The following example illustrates the above results.

Example 4. Consider the matrices $A_{3}\left(\frac{1}{6},-\frac{1}{3}\right)$ and $B(1)$ given in Example 3. Now consider the perturbation $\Delta=\left(\begin{array}{lll}0 & 0 & \frac{1}{10}\end{array}\right)$. Then $\sigma\left(\widetilde{A}_{3}\right)=\{0.666,-0.282,0.216\}$. Note that all the eigenvalues have been 
perturbed as established in Proposition 8. Moreover, the matrix $\widetilde{A}_{3}$ is stable, since $\widetilde{\rho}<1$. This fact is in according to Proposition 9, since $0.1=\left\|\Delta_{2}\right\|_{F}<\frac{1-\rho}{|d|}=1-0.638$.

\section{Conclusions}

A structured system obtained applying the finite-difference method to a partial differential equation is considered. This system is a structured system with unknown parameters, where the state coefficient matrix is tridiagonal. Robust stability of the state coefficient matrix affected by different perturbations is studied using the Frobenius and infinity matrix norms. Results on the characteristic polynomial and eigenvalues of the matrices involved in the problem are developed to assure the robustness of stability. Some robust sufficient stability conditions for structured discrete-time system are presented in terms of the initial parameters of the model. It is interesting that, in contrast to the bounds given by other authors, the determination of bound given in this paper does not depend on complex calculations. This bound can be calculated directly from the initial coefficient matrices. This means it can be used for the design of algorithms for solving engineering problems where robust control is critical.

Acknowledgment This research is supported by Grant MTM201018228.

\section{References}

[1] K. Zhow, J. Doyle, K.Glover, Robust and Optimal Control, Prentice Hall, New Jersey, 1996.

[2] D. Hinrichsen, A.J. Pritchard, Real and complex stability radii: a survey. Control of Uncertain Systems, in: Progress in System and Control Theory 6, 1990, 119-162.

[3] D. Hinrichsen, N.K. Son, Stability radii of linear discrete-time systems and symplectic pencils, International Journal Robust and Nonlinear Control 1 (1991) 79-97.

[4] D. Hinrichsen, N. K. Son, P. H. Ngoc, Stability radii of higher order positive difference systems, Systems and Control Letters 49 (5) (2003) 377-388. 
[5] S. H. Chen, J. H. Chou, L. A. Zheng, Stability robustness of linear output feedback systems with both time-varying structured and unstructured parameter uncertainties as well as delayed perturbations, Journal of the Franklin Institute 342 (2005) 213-234.

[6] K. Zhou, P.P. Khargonekar, Stability robustness bounds for linear state-space models with structured uncertainty, IEEE Transactions on Automatic Control AC-32 (7) (1987) 621-623.

[7] S.J. Chen, J.L. Lin, Robust D-stability of discrete and continuous time interval systems, Journal of the Franklin Institute 341 (2004) 505-517.

[8] S. A. He, I. K. Fong, Output stabilization of a class of secondorder linear time-delay systems: An eigenvalue approach, Journal of the Franklin Institute 349 (2011) 110-127.

[9] M. C. de Oliveira, J. C. Geromel, L. Hsu, LMI characterization of structural and robust stability: the discrete-time case, Linear Algebra and its Applications 296 (1999) 27-38.

[10] M. C. de Oliveira, J. Bernussou, J. C. Geromel, A new discretetime robust stability condition, Systems and Control Letters 37 (1999) 261-265.

[11] B. Cantó, C. Coll, E. Sánchez, Identifiability for a Class of Discretized Linear Partial Differential Algebraic Equations, Mathematical Problems in Engineering (2011) 1-12.

[12] A. K. Jain, Advances in Mathematical Models for Image Processing, Proceedings of the IEEE 69(5) (1981) 502-528.

[13] R. Bhatia, Matrix Analysis, Springer-Verlag, New York, 1997.

[14] B. Cantó, C. Coll, E. Sánchez, Structural identifiability of a model of dialysis, Mathematical and Computer Modelling 50 (2009) 733737.

[15] G.H. Golub, C.F. Van Loan in: Matrix Computations, The Johns Hopkins University Press, Baltimore, 1996.

[16] J.J.M. Cuppen, A divide and conquer method for the symmetric tridiagonal eigenproblem, Numerische Mathematik 36 (1981) 177195. 